\title{
Local holomorphic De Rham cohomology
}

\author{
Rong Du and Stephen Yau
}

Local holomorphic De Rham cohomology introduced in this paper and punctured local holomorphic De Rham cohomology introduced by Huang-Luk-Yau are two important local invariants for varieties with isolated singularities. We find some relations between these two invariants and the invariants defined by Steenbrink on surface singularities, and from which we use these two invariants to describe when a Gorenstein singularity is quasi-homogeneous.

\section{Introduction}

Let $M$ be a complex manifold. The $q$ th holomorphic De Rham cohomology of $M, H_{h}^{q}(M)$, is defined to be the $d$-closed holomorphic $q$-forms modulo the $d$ exact holomorphic $q$-forms. Holomorphic De Rham cohomology was studied by Hörmander [4]. In the paper [5], the authors introduce the notion of $q$ th punctured local holomorphic De Rham cohomology $H_{h}^{q}(V, x)$ as the direct limit of $H_{h}^{q}(U-\{x\})$ where $U$ runs over strongly pseudoconvex neighborhoods of isolated singularity $x$ in a complex variety $V$. This is an important local invariant which can be used to tell when the hypersurface singularity is quasi-homogeneous (see Section 2). The purpose of this paper is to generalize the result in [5] to Gorenstein surface singularities, i.e., the following theorem.

Main Theorem. Let $(V, x)$ be an isolated Gorenstein surface singularity. For a given smoothing, let $\mu$ and $\tau$ be the generalized Milnor number and Tjurina number of $(V, x)$, respectively. Then

$$
\operatorname{dim} H_{h}^{2}(V, x)-\operatorname{dim} H_{h}^{1}(V, x)=\mu-\tau
$$

In particular, the singularity is quasi-homogeneous if and only if

$$
\operatorname{dim} H_{h}^{1}(V, x)=\operatorname{dim} H_{h}^{2}(V, x) .
$$


In Section 2, we recall the basic notions and results that are needed for the proof of our main theorem. We also introduce the notion local holomorphic De Rham cohomology. In Section 3, we establish some relations between our new invariant and the invariants defined by Steenbrink. We also prove our main theorem in this section.

\section{Preliminary}

Let $V$ be an $n$-dimensional complex analytic subvariety in $\mathbb{C}^{N}$ with only isolated singularities. There are two kinds of sheaves of germs of holomorphic $p$-forms defined on $V$ (cf.[12]):

(1) $\bar{\Omega}_{V}^{p}:=\pi_{*} \Omega_{M}^{p}$, where $\pi: M \longrightarrow V$ is a resolution of singularities of $V$.

(2) $\overline{\bar{\Omega}}_{V}^{p}:=\theta_{*} \Omega_{V \backslash V_{\text {sing }}}^{p}$ where $\theta: V \backslash V_{\text {sing }} \longrightarrow V$ is the inclusion map and $V_{\text {sing }}$ is the singular set of $V$.

Clearly $\bar{\Omega}_{V}^{p}$ is a coherent sheaf because $\pi$ is a proper map. $\overline{\bar{\Omega}}_{V}^{p}$ is also a coherent sheaf by a Theorem of Siu [7]. In case $V$ is a normal variety, the dualizing sheaf $\omega_{V}$ of Grothendieck is actually the sheaf $\overline{\bar{\Omega}}_{V}^{n}$.

Definition 2.1. The Siu complex is a complex of coherent sheaves $J^{\bullet}$ supported on the singular points of $V$ which is defined by the following exact sequence:

$$
0 \longrightarrow \bar{\Omega}^{\bullet} \longrightarrow \overline{\bar{\Omega}}^{\bullet} \longrightarrow J^{\bullet} \longrightarrow 0
$$

Definition 2.2. Let $V$ be an $n$-dimensional Stein space with $x$ as its only singular point. Let $\pi:(M, A) \rightarrow(V, x)$ be a resolution of the singularity with $A$ as exceptional set. The geometric genus $p_{\mathrm{g}}$ and the irregularity $q$ of the singularity are defined as follows (cf.[8, 13]):

$$
\begin{aligned}
p_{\mathrm{g}} & :=\operatorname{dim} \Gamma\left(M \backslash A, \Omega^{n}\right) / \Gamma\left(M, \Omega^{n}\right), \\
q & :=\operatorname{dim} \Gamma\left(M \backslash A, \Omega^{n-1}\right) / \Gamma\left(M, \Omega^{n-1}\right),
\end{aligned}
$$

The $s$-invariant of the singularity is defined as follows:

$$
s:=\operatorname{dim} \Gamma\left(M \backslash A, \Omega^{n}\right) /\left[\Gamma\left(M, \Omega^{n}\right)+d \Gamma\left(M \backslash A, \Omega^{n-1}\right)\right] .
$$

Lemma 2.1 [6]. Let $V$ be an n-dimensional Stein space with $x$ as its only singular point. Let $J^{\bullet}$ be the Siu complex of coherent sheaves supported on $x$. Then 
(1) $\operatorname{dim} J^{n}=p_{\mathrm{g}}$

(2) $\operatorname{dim} J^{n-1}=q$,

(3) $\operatorname{dim} J^{i}=0$, for $1 \leq i \leq n-2$,

(4) $s=\operatorname{dim} H^{n}\left(J^{\bullet}\right)=p_{\mathrm{g}}-q$ and $\operatorname{dim} H^{n-1}\left(J^{\bullet}\right)=0$.

In analogous to the definition of the $q$ th punctured local holomorphic De Rham cohomology, we can similarly define local holomorphic De Rham cohomology $H_{h}^{q}(V)_{x}$ to be the direct limit of $H_{h}^{q}(U)$ where $U$ runs over strongly pseudoconvex neighborhoods of $x$ in $V$.

Definition 2.3. Let $x$ be a singularity of $V$. Consider the complexes

$$
\begin{aligned}
& 0 \longrightarrow \mathbb{C} \longrightarrow \mathscr{O}_{V, x} \stackrel{\bar{d}^{0}}{\longrightarrow} \bar{\Omega}_{V, x}^{1} \stackrel{\bar{d}^{1}}{\longrightarrow} \bar{\Omega}_{V, x}^{2} \stackrel{d^{2}}{\longrightarrow} \cdots \\
& 0 \longrightarrow \mathbb{C} \longrightarrow \mathscr{O}_{V, x} \stackrel{\overline{\bar{d}}^{0}}{\longrightarrow} \overline{\bar{\Omega}}_{V, x}^{1} \stackrel{\overline{\bar{d}}^{1}}{\longrightarrow} \overline{\bar{\Omega}}_{V, x}^{2} \stackrel{\overline{\bar{d}}^{2}}{\longrightarrow} \cdots
\end{aligned}
$$

Then the generalized Poincaré number $\bar{p}_{x}^{(i)}, \overline{\bar{p}}_{x}^{(i)}$ are defined by dim $\operatorname{ker} \bar{d}^{i} / \operatorname{Im} \bar{d}^{i-1}$ and $\operatorname{dim} \operatorname{ker} \overline{\bar{d}}^{i} / \operatorname{Im} \overline{\bar{d}}^{i-1}$, respectively.

Lemma 2.2. Let $(V, x)$ be an isolated singularity. The the local holomorphic De Rham cohomology $H_{h}^{q}(V)_{x}$ and punctured local holomorphic De Rham cohomology $H_{h}^{q}(V, x)$ are isomorphic to qth cohomology of the following complex:

$$
0 \longrightarrow \mathbb{C} \longrightarrow \mathscr{O}_{V, x} \stackrel{\bar{d}^{0}}{\rightarrow} \bar{\Omega}_{V, x}^{1} \stackrel{\bar{d}^{1}}{\rightarrow} \bar{\Omega}_{V, x}^{2} \stackrel{d^{2}}{\rightarrow} \cdots
$$

and

$$
0 \longrightarrow \mathbb{C} \longrightarrow \mathscr{O}_{V, x} \stackrel{\overline{\bar{d}}^{0}}{\rightarrow} \overline{\bar{\Omega}}_{V, x}^{1} \stackrel{\overline{\bar{d}}^{1}}{\rightarrow} \overline{\bar{\Omega}}_{V, x}^{2} \stackrel{\overline{\bar{d}}^{2}}{\rightarrow} \cdots
$$

respectively.

The main theorem in [5] is

Theorem 2.1 [5]. Let $(V, x)=\left\{\left(z_{0}, \ldots, z_{n}\right) \in \mathbb{C}^{n+1}: f\left(z_{0}, \ldots, z_{n}\right)=0\right\}$ be a hypersurface with origin as isolated singularity. Then

(1) $\operatorname{dim} H_{h}^{q}(V, x)=0$ for $q \leq n-2$, 
(2) $\operatorname{dim} H_{h}^{n}(V, x)-\operatorname{dim} H_{h}^{n-1}(V, x)=\mu-\tau$, where

$$
\mu=\operatorname{dim} \mathbb{C}\left\{z_{0}, \ldots, z_{n}\right\} /\left(\partial f / \partial z_{0}, \ldots, \partial f / \partial z_{n}\right)
$$

is the Milnor number and

$$
\tau=\operatorname{dim} \mathbb{C}\left\{z_{0}, \ldots, z_{n}\right\} /\left(f, \partial f / \partial z_{0}, \ldots, \partial f / \partial z_{n}\right)
$$

is the Tjurina number of the singularity $(V, x)$, respectively.

For isolated surface singularities, we can generalize above theorem to Gorenstein case. Before doing that, we recall some basic notions.

Let $(V, x)$ be a normal surface singularity. Let $\pi: M \longrightarrow V$ be a good resolution of singularity. Let $\pi^{-1}(x)=A=\cup A_{i}, 1 \leq i \leq n$, be the irreducible decomposition of the exceptional set $A$ into irreducible components. Let $g_{i}$ be the genus of $A_{i}, g=\sum g_{i}$ and denote by $\widetilde{A}$ the disjoint union of $A_{i}$. Let $\Gamma$ be the dual graph of $A$ and $b$ is the number of loops in $\Gamma$.

The sheaf of germs of logarithmic one-forms $\Omega_{M}^{1}(\log A)$ is defined by the kernel of the restriction map.

$$
0 \longrightarrow \Omega_{M}^{1}(\log A)(-A) \longrightarrow \Omega_{M}^{1} \longrightarrow \Omega_{\widetilde{A}}^{1} \longrightarrow 0
$$

It follows that $\wedge^{2} \Omega_{M}^{1}(\log A)=\Omega_{M}^{2}(A)$, and there is an exact sequence

$$
0 \longrightarrow \Omega_{M}^{1} \longrightarrow \Omega_{M}^{1}(\log A) \longrightarrow \mathscr{O}_{\widetilde{A}}^{1} \longrightarrow 0 .
$$

The following lemma can be found in [10].

Lemma 2.3. (1) The composition $H^{0}\left(\mathscr{O}_{\widetilde{A}}\right) \rightarrow H^{1}\left(\Omega_{M}^{1}\right) \rightarrow H^{1}\left(\Omega_{\widetilde{A}}^{1}\right)$ is an isomorphism.

(2) $H^{0}\left(\Omega_{M}^{1}\right) \widetilde{\rightarrow} H^{0}\left(\Omega_{M}^{1}(\log A)\right)$.

Recall that $n$ is the number of the components of $A$.

Since $H_{A}^{0}\left(\mathscr{O}_{\widetilde{A}}\right) \widetilde{\rightarrow} H^{0}\left(\mathscr{O}_{\widetilde{A}}\right)$, the map $H^{0}\left(\mathscr{O}_{\widetilde{A}}\right) \rightarrow H^{1}\left(\Omega_{M}^{1}\right)$ factors via $H_{A}^{1}\left(\Omega_{M}^{1}\right)$. Therefore, by Lemma 2.3, Steenbrink define an nonnegative integer

$$
\gamma:=\operatorname{rk}\left(H_{A}^{1}\left(\Omega_{M}^{1}\right) \rightarrow H^{1}\left(\Omega_{M}^{1}\right)\right)-n .
$$

Besides $\gamma$, Steenbrink introduces two other invariants

$$
\begin{aligned}
\alpha & :=\operatorname{dim} H^{0}\left(\Omega_{M}^{2}\right) / d H^{0}\left(\Omega_{M}^{1}(\log A)(-A)\right), \\
\beta & :=\operatorname{dim} H^{0}\left(\Omega_{\widetilde{A}}^{1}\right) / \operatorname{Im} H^{0}\left(\Omega_{M}^{1}\right) .
\end{aligned}
$$


Next let us recall the definition of smoothable singularities, generalized Milnor number and Tjurina number.

Definition 2.4. If there exists a flat morphism $\pi: \mathcal{V} \rightarrow T$ of local analytic spaces such that $\pi^{-1}\left(t_{0}\right) \simeq V$ and $\pi^{-1}(t) \simeq V_{t}$ is nonsingular for $t \neq t_{0}$, then the singularity $(V, x)$ is called smoothable.

More generally, let $(V, x)$ be a local analytic variety with isolated singularity of pure dimension $n$. To any smoothing $\pi: \mathcal{V} \rightarrow T$ of $V$, one can attach a Milnor fibre $F:=B_{\epsilon} \cap \pi^{-1}(t)$, where $B_{\epsilon}$ is a ball in some $\mathbb{C}^{N}$ containing $\mathcal{V}$ and $t \in T$ (cf.[9]). $F$ is a $2 n$-manifold with boundary, with the homotopy type of a finite complex of dimension $n$. We define Milnor number $\mu=\operatorname{rk} H_{n}(F)$.

As is well known, any complex analytic germ $(V, x)$ with isolated singularity admits a semi-universal deformation

$$
(V, x) \hookrightarrow(\mathscr{Y}, y) \stackrel{F}{\rightarrow}(S, s)
$$

(cf.[1, 2]). Given a semi-universal deformation of $(V, x)$, we call an irreducible component $\left(S^{\prime}, s\right)$ of $(S, s)$ a smooth component if the general fiber of $F$ over this component is smooth. In general, however, the germ $(S, s)$ is not smooth and indeed, it may have irreducible components of various dimensions. Any smooth component has dimension between $\operatorname{dim}_{\mathbb{C}} T_{V}^{1}-\operatorname{dim}_{\mathbb{C}} T_{V}^{2}$ and $\operatorname{dim}_{\mathbb{C}} T_{V}^{1}$, where $T_{V}^{1}, T_{V}^{2}$ are, respectively, the first order deformation and obstruction spaces.

For a smoothing, $\pi: \mathcal{V} \rightarrow T$ of $(V, x)$ and a smooth component $\left(S^{\prime}, s\right)$ on which $\pi: \mathcal{V} \rightarrow T$ lies, Wahl had a conjecture in [11]:

$$
\operatorname{dim}_{\mathbb{C}}\left(S^{\prime}, s\right)=\operatorname{dim}_{\mathbb{C}} \operatorname{Coker}\left(\Theta_{\mathcal{V} / T} \otimes \mathcal{O}_{V} \rightarrow \Theta_{V}\right)
$$

Wahl himself verified his conjecture for special cases. Later Greuel and Looijenga [3] proved this conjecture completely.

We define $\tau=\operatorname{dim}_{\mathbb{C}} T_{V}^{1}$. If $(V, x)$ has no obstructed deformations (e.g., $(V, x)$ is a complete intersection), then $(S, s)$ is nonsingular and

$$
\operatorname{dim}_{\mathbb{C}}(S, s)=\operatorname{dim}_{\mathbb{C}} T_{V}^{1}
$$

So $\tau$ generalizes the usual Tjurina number. 


\section{Proof of Main Theorem}

Denote

$h_{h}^{i}(M)=\operatorname{dim} H_{h}^{i}(M), h^{i}(M, \mathscr{F})=\operatorname{dim} H^{i}(M, \mathscr{F}), h^{i}(M)=\operatorname{dim} H^{i}(M, \mathbb{C})$,

where $M$ is a manifold, $\mathscr{F}$ is a sheaf on $M$.

Proposition 3.1. Let $(V, x)$ be a normal surface singularity. Then

$$
\operatorname{dim} H_{h}^{2}(V)_{x}-\operatorname{dim} H_{h}^{1}(V)_{x}=\alpha+\beta-g .
$$

Proof. Let $\pi: M \longrightarrow V$ be a good resolution of singularity. Let $\pi^{-1}(x)=$ $A=\cup A_{i}, 1 \leq i \leq n$, be the irreducible decomposition of the exceptional set $A$ into irreducible components. Since $\operatorname{dim} H_{h}^{2}(V)_{x}$ and $\operatorname{dim} H_{h}^{1}(V)_{x}$ are local invariants, we can suppose that $V$ is sufficiently small and is contractible to the singular point without loss of generality.

We have the spectral sequence

$$
E_{1}^{p, q}=H^{q}\left(M, \Omega_{M}^{p}\right) \Rightarrow \mathbf{H}^{p+q}\left(M, \Omega_{M}^{\bullet}\right) \cong H^{p+q}(M, \mathbb{C}) .
$$

The spectral sequence induces an exact sequence of small order terms

$$
0 \rightarrow H_{h}^{1}(M) \rightarrow H^{1}(M, \mathbb{C}) \rightarrow E_{2}^{0,1} \rightarrow H_{h}^{2}(M) \rightarrow H^{2}(M, \mathbb{C}) \rightarrow E_{2}^{1,1} \rightarrow 0,
$$

where

$$
\begin{gathered}
E_{2}^{0,1}=\operatorname{ker}\left(H^{1}\left(M, \mathscr{O}_{M}\right) \rightarrow H^{1}\left(M, \Omega_{M}^{1}\right)\right), \\
E_{2}^{1,1}=\operatorname{coker}\left(H^{1}\left(M, \mathscr{O}_{M}\right) \rightarrow H^{1}\left(M, \Omega_{M}^{1}\right)\right) .
\end{gathered}
$$

So

$$
h_{h}^{1}(M)-h^{1}(M)+\operatorname{dim} E_{2}^{0,1}-h_{h}^{2}(M)+h^{2}(M)-\operatorname{dim} E_{2}^{1,1}=0 .
$$

Since

$$
\operatorname{dim} E_{2}^{0,1}-\operatorname{dim} E_{2}^{1,1}=h^{1}\left(M, \mathscr{O}_{M}\right)-h^{1}\left(M, \Omega_{M}^{1}\right),
$$

we have

$$
h_{h}^{1}(M)-h^{1}(M)-h_{h}^{2}(M)+h^{2}(M)+h^{1}\left(M, \mathscr{O}_{M}\right)-h^{1}\left(M, \Omega_{M}^{1}\right)=0
$$


From [10], we know

$$
\begin{aligned}
h^{1}\left(M, \Omega_{M}^{1}\right) & =\gamma+q+n \\
& =p_{\mathrm{g}}-g-b-\alpha-\beta+n .
\end{aligned}
$$

And

$$
h^{1}(M)=\operatorname{dim} H^{1}(A, \mathbb{C})=2 g+b, \quad h^{2}(M)=n, \quad h^{1}\left(M, \mathscr{O}_{M}\right)=p_{\mathrm{g}} .
$$

So

$$
h_{h}^{2}(M)-h_{h}^{1}(M)=\alpha+\beta-g .
$$

Since $\operatorname{dim} H_{h}^{2}(V)_{x}$ and $\operatorname{dim} H_{h}^{1}(V)_{x}$ are independent on the choice of the pseudoconvex domains containing the singularity,

$$
\begin{aligned}
\operatorname{dim} H_{h}^{2}(V)_{x}-\operatorname{dim} H_{h}^{1}(V)_{x} & =\bar{p}_{x}^{(2)}-\bar{p}_{x}^{(1)} \\
& =h_{h}^{2}(M)-h_{h}^{1}(M) \\
& =\alpha+\beta-g .
\end{aligned}
$$

Proposition 3.2. Let $(V, x)$ be an isolated normal surface singularity. Then

$$
\overline{\bar{p}}_{x}^{(1)}=\bar{p}_{x}^{(1)}, \quad \overline{\bar{p}}_{x}^{(2)}=\bar{p}_{x}^{(2)}+s .
$$

Proof. Let $\pi: M \longrightarrow V$ be a good resolution of singularity. Let $\pi^{-1}(x)=A$, be the exceptional set. Since $\operatorname{dim} H_{h}^{i}(V)_{x}$ and $H_{h}^{i}(V, x), i=1,2$, are local invariants, we can suppose that $V$ is sufficiently small and is contractible to the singular point without loss of generality.

According to the short exact sequence (2.1) and Cartan Theorem A, we have the following exact sequence of complexes:

$$
0 \longrightarrow \Gamma\left(V, \bar{\Omega}^{\bullet}\right) \longrightarrow \Gamma\left(V, \overline{\bar{\Omega}}^{\bullet}\right) \longrightarrow \Gamma\left(V, J^{\bullet}\right) \longrightarrow 0
$$

In view of Lemma 2.1(4), (3.10) gives

$$
\begin{array}{r}
0 \longrightarrow H^{1}\left(\Gamma\left(V, \bar{\Omega}^{\bullet}\right)\right) \longrightarrow H^{1}\left(\Gamma\left(V, \overline{\bar{\Omega}}^{\bullet}\right)\right) \longrightarrow H^{1}\left(\Gamma\left(V, J^{\bullet}\right)\right) \\
\longrightarrow H^{2}\left(\Gamma\left(V, \bar{\Omega}^{\bullet}\right)\right) \longrightarrow H^{2}\left(\Gamma\left(V, \overline{\bar{\Omega}}^{\bullet}\right)\right) \longrightarrow H^{2}\left(\Gamma\left(V, J^{\bullet}\right)\right) \longrightarrow 0 .
\end{array}
$$

Since $J^{\bullet}$ is supported on $x$, we have

$$
H^{2}\left(\Gamma\left(V, J^{\bullet}\right)\right)=H^{2}\left(J^{\bullet}\right), \quad H^{1}\left(\Gamma\left(V, J^{\bullet}\right)\right)=H^{1}\left(J^{\bullet}\right) .
$$


So from Lemma 2.1(4), we get

$$
H_{h}^{1}(M) \cong H_{h}^{1}(M \backslash A)
$$

and

$$
0 \longrightarrow H^{2}\left(\Gamma\left(V, \bar{\Omega}^{\bullet}\right)\right) \longrightarrow H^{2}\left(\Gamma\left(V, \overline{\bar{\Omega}}^{\bullet}\right)\right) \longrightarrow H^{2}\left(\Gamma\left(V, J^{\bullet}\right)\right) \longrightarrow 0
$$

Observe that

$$
\begin{gathered}
H^{2}\left(\Gamma\left(V, \bar{\Omega}^{\bullet}\right)\right)=H^{2}\left(\Gamma\left(M, \Omega_{M}^{\bullet}\right)\right)=H_{h}^{2}(M), \\
H^{2}\left(\Gamma\left(V, \bar{\Omega}^{\bullet}\right)\right)=H^{2}\left(\Gamma\left(M \backslash A, \Omega_{M}^{\bullet}\right)\right)=H_{h}^{2}(M \backslash A) .
\end{gathered}
$$

It follows from (3.13) and Lemma 2.1(4) that

$$
h_{h}^{2}(M \backslash A)=h_{h}^{2}(M)+s .
$$

Since $\operatorname{dim} H_{h}^{i}(V)_{x}$ and $\operatorname{dim} H_{h}^{i}(V, x), i=1,2$, are independent on the choice of the pseudoconvex domains containing the singularity,

$$
\overline{\bar{p}}_{x}^{(1)}=\bar{p}_{x}^{(1)}, \quad \overline{\bar{p}}_{x}^{(2)}=\bar{p}_{x}^{(2)}+s .
$$

Proposition 3.3. Let $(V, x)$ be an isolated normal surface singularity. Then

$$
\operatorname{dim} H_{h}^{2}(V, x)-\operatorname{dim} H_{h}^{1}(V, x)=\overline{\bar{p}}_{x}^{(2)}-\overline{\bar{p}}_{x}^{(1)}=2 \alpha+2 \beta+\gamma+b \geq 0 .
$$

Proof. From Propositions 3.1 and 3.2, and

$$
s=\alpha+\beta+\gamma+g+b
$$

from Steenbrink (cf.[10]), we can get the result.

Theorem 3.1 [10]. Let $(V, x)$ be an isolated Gorenstein surface singularity. For a given smoothing, let $\mu$ and $\tau$ be the generalized Milnor number and Tjurina number of $(V, x)$, respectively. Then

$$
\mu-\tau=2 \alpha+2 \beta+\gamma+b
$$


Theorem 3.2. Let $(V, x)$ be an isolated Gorenstein surface singularity. For a given smoothing, let $\mu$ and $\tau$ be the generalized Milnor number and Tjurina number of $(V, x)$, respectively. Then

$$
\operatorname{dim} H_{h}^{2}(V, x)-\operatorname{dim} H_{h}^{1}(V, x)=\mu-\tau
$$

In particular, the singularity is quasi-homogeneous if and only if

$$
\operatorname{dim} H_{h}^{1}(V, x)=\operatorname{dim} H_{h}^{2}(V, x) .
$$

Proof. The theorem follows from Proposition 3.3 and Theorem 3.1.

\section{Acknowledgments}

S.A. was partially supported by Institute of Mathematics, East China Normal University, Shanghai, China and research was partially supported by NSF. F.A. was supported by NSFC and PSSCS of Shanghai.

\section{References}

[1] J. Bingener, Offenheit der Versalität in der analytischen Geometrie, Math. Z. 173 (1980), 241-281.

[2] H. Grauert, Über die Deformation isolierter Singularitäten analytischer Mengen, Invent. Math. 15 (1972), 171-198.

[3] G.-M. Greuel and E. Looijenga, The dimension of smoothing components, Duke Math. J. 52(1) (1985), 263-272.

[4] L. Hörmander, An introduction to complex analysis in several complex variables, Van Nostrand, Princeton, 1996.

[5] X.J. Huang, H.S. Luk and S.S.-T. Yau, Punctured local holomorphic De Rham cohomology, J. Math. Soc. Japan 55(3) (2003), 633-640.

[6] H.S. Luk and S.S.-T. Yau, Kohn-Rossi cohomology and its application to the complex Plateau problem, II, J. Differential Geom. 77 (2007), $135-148$.

[7] Y.-T. Siu, Analytic sheaves of local cohomology, Trans. AMS 148 (1970), 347-366. 
[8] D.V. Straten and J. Steenbrink, Extendability of holomorphic differential forms near isolated hypersurface singularities, Abh. Math. Sem. Univ. Ham- burg 55 (1985), 97-110.

[9] Lê Dũng Tráng: Some remarks on relative monodromy, Proceedings of the Nordic Summer School. Sijthoff and Noordhoff, Oslo, 1976.

[10] J. Wahl, A characterization of quasi-homogeneous Gorenstein surface singu-larities, Compositio Math. 55 (1985), 269-288.

[11] J. Wahl, Smoothings of normal surface singularities, Topology 20 (1981), 219-246.

[12] S.S.-T. Yau, Various numerical invariants for isolated singularities, Amer. J. Math. 104(5) (1982), 1063-1110.

[13] S.S.-T. Yau, Existence of $L^{2}$-integrable holomorphic forms and low estimates of $T_{V}^{1}$, Duke Mthe. J. 48(3) (1981), 537-547.

Department of Mathematics

East China Normal University

No. 500, Dongchuan Road

Shanghai, 200241, P. R. China

E-mail address: discreetwhy@hotmail.com

Department of Mathematics

Statistics and Computer Science

UNiversity OF Illinois at ChicAgo

851 S. Morgan Street

Chicago, IL

60607-7045, USA

E-mail address: rdu2@uic.edu

E-mail address: yau@uic.edu

Received September 30, 2009 